demerec, M., and hartman, P. E. 1959. Complex loci in Microorganisms. Ann. Rev. Microbiol., 13, 377-406.

DE SERRES, F. J. 1963. Studies with purple adenine mutants in Neurospora crassa. V. Evidence for allelic complementation among ad-3B mutants. Genetics, 48, 351-360.

FINCHAM, J. R. S., AND CODdington, A. 1963. The Mechanism of Complementation between am mutants of Neurospora crassa. Cold Spring Harb. Symp. Quant. Biol., 28, 517-527.

GAREN, A., AND GAREN, s. 1963. Complementation in vivo between structural mutants of alkaline phosphatase from E. coli. 7. Mol. Biol., 7, 13-22.

grles, N. H. 1958. Mutations at specific loci in Neurospora. Proc. Xth Int. Cong. Gen., 1, 261-279.

KAPlan, S., suYAmA, Y., AND BONNER, D. M. 1964. Fine structure analysis at the td locus of Neurospora crassa. Genetics, 49, 145-158.

LACY, A. M. 1965. Structural and physiological relationships within the td locus in Neurospora crassa. Biochem. Biophys. Res. Comm., 18, 812-823.

LACY, A. M., AND BONNER, D. м. 1961. Complementation between alleles of the td locus in Neurospora crassa. Proc. Natl. Acad. Sci. U.S., 47, 72-77.

SChlesinger, M. J., AND levinthal, c. 1963. Hybrid protein formation of $E$. coli alkaline phosphatase leading to in vitro complementation. f. Mol. Biol., 7, 1-12.

SUYAMA, Y., LACY, A. M., BONNER, D. M. 1964. A genetic map of the td locus of Neurospora crassa. Genetics, 49, 135-144.

voGel, H. J. 1956. A convenient growth medium for Neurospora (Medium N.). Microb. genet. Bull., 13, 42-43.

\title{
SPERM UTILISATION AND RADIATION SENSITIVITY IN DROSOPHILA MELANOGASTER
}

\author{
B. M. SLIZYNSKI \\ Mutagenesis Research Unit of the M.R.C. Institute of Animal Genetics, Edinburgh University
}

Received 10.vi.69

\section{INTRODUCTION}

IN a study of X-ray induced structural changes in Drosophila melanogaster Slizynska (1963) found heterogeneity among males in their response to the treatment. She suggested that this heterogeneity may be due to the differences in the proportion of spermatogonia that were in a sensitive stage at the time of irradiation. High sensitivity would thus be caused by a high proportion of sensitive cells. A change in the proportion of cells in any stage of spermatogenesis may affect the number of spermatozoa produced per unit of time. Thus more offspring would be produced per unit of time when cell cycles of spermatogenesis were shortened and vice-versa.

Many experiments on mutagenesis have been carried out using mass cultures or pooling the results from many males; this naturally obscured all differences between males. The present experiment was planned to investigate whether there are any natural differences among males in their production of spermatozoa (measured by the number of offspring) in a limited time of 10 days.

\section{Material AND methods}

One hundred one-day-old Ore-K males were given each a batch of four one-day-old virgin females every other day. There were 5 such batches of females in the course of the experiment, thus each male had an opportunity to fertilise 20 females in the period of 10 days. The offspring of females were 
scored till no more of them appeared in the vial. The food was standard (corn-mollasses-agar-yeast) used in this laboratory and the flies were kept at $+25^{\circ} \mathrm{C}$.

\section{Results}

After discarding the incomplete records, the following results can be presented. In all, 63 males and 1260 females, of which 273 were not fertilised, yielded altogether 50,220 flies. Males had on the average $797 \pm 28$ (331-1301) and females $51 \pm 2$ (1-146) offspring. On the basis of the number and distribution of offspring among successive batches, the males can be divided into three groups: A, B and C. This is shown in the table $\mathrm{l}$ and in the diagram fig. 1 .

\section{TABLE 1}

Some numerical characteristics of the three types of Drosophila melanogaster males

\begin{tabular}{lrrrr}
\multicolumn{1}{c}{ Number of } & Type A & Type B & Type C & A+B + C \\
Males & 9 & 40 & 14 & 63 \\
Females & 180 & 800 & 280 & 1,260 \\
Offspring & 8,221 & 33,788 & 8,111 & 50,220 \\
per male & $913 \pm 39$ & $845 \pm 12$ & $579 \pm 15$ & $797 \pm 28$ \\
in the 1st batch & $3,076=100$ & $8,235=100$ & $2,426=100$ & $13,737=100$ \\
2nd batch & 72 & 99 & 77 & 99 \\
3rd batch & 51 & 98 & 21 & 84 \\
4th batch & 33 & 37 & - & 23 \\
5th batch & 11 & 35 & &
\end{tabular}

In group $\mathrm{A}$, there is a steady and uniform fall in the number of offspring, the highest yield being in the first batch. The production in this group is the highest: 46 offspring per male per day.

In group B, the number of offspring remains rather constant with some falling off towards the end. The highest yield is in the first batch although the yield of the second batch can sometimes approach or even exceed it. The males of this group gave on the average 42 offspring per day.

Group $\mathrm{C}$ contains males which within the 10 days' limit have short period of fertility, the highest yield being in the second batch. The production in this group is the lowest: 29 offspring per male per day. There is a rapid decline of the number of offspring in the last batch. No male of this group had any offspring in the fifth batch of females.

Statistical treatment of the results is concerned with the solving of the question whether the collection of males defined by their individual fertilities represents one or more than one population. The method applied here was introduced by Czekanowski in 1913, and is particularly suited to the problem. It consists of ( 1 ) obtaining mean differences (disregarding the + and sign) between all members of the sample; (2) arranging the differences in an increasing order for each member of the sample and (3) placing the least different members of the sample near each other in the diagram. The calculating sheet shown in table 2 illustrates the method.

The results in a form of a diagram show the similarity relations between all members of the sample. In the diagram the differences are graded: the first lowest three differences are marked by black squares, the next two by squares with several vertical lines and the sixth difference by a square with one vertical line. All higher differences are left as blank spaces. 
The results based on the data of 27 males (nine from each group) shown in the form of a diagram (fig. 1) clearly demonstrate the existence of three not similar groups of males.

At the intersection of the vertical column marked on the diagram with letter -a- with the horizontal row marked -a- is the group A of males, and at the intersection of column a with row $\mathrm{c}$ is the area of relation of group A with group C, and so on. Absence of low differences in the area of relation indicates the lack of similarity between groups.

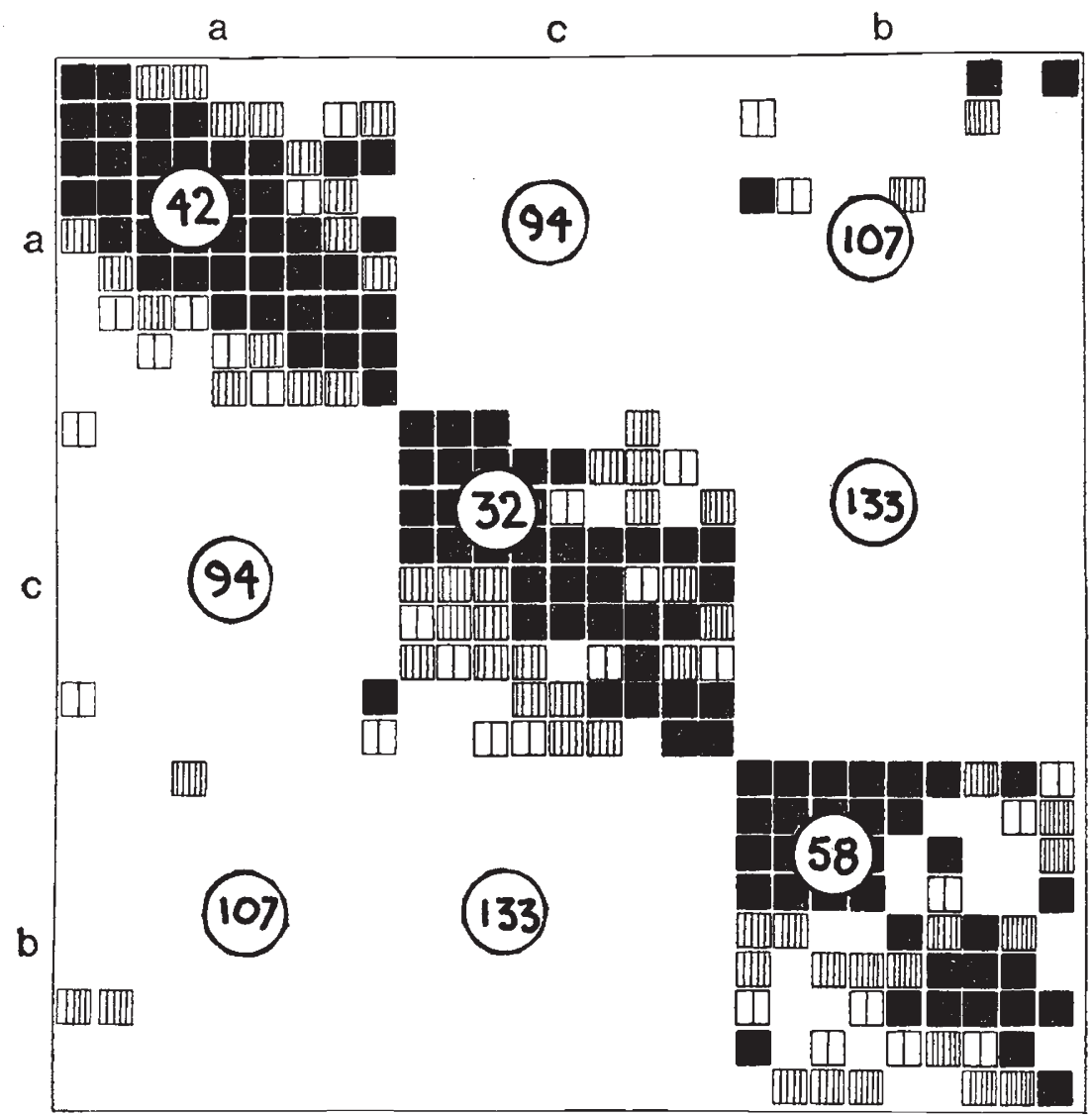

FIG. 1.-Diagram of the results of Czekanowski's differential diagnosis of the collection of 27 males analysed for the production of spermatozoa. There are three distinct groups: top left-group A, bottom right-group B and in the middle group C. The side connections in the upper right between group $\mathrm{A}$ and $\mathrm{B}$ may be due either to accidental causes or to the imperfection of the present arrangement. (For details see text.)

The results of Czekanowski's analysis as shown in the diagram may be expressed by mean differences within the groups and between them. The smaller the intra-group difference the more compact is the group and conversely the larger the inter-group difference the less similar are the groups in question. The mean differences (round figures of which are inserted in appropriate places in the diagram) are: for group $\mathrm{A}=41 \cdot 9 \pm 2 \cdot 7$, for group $A$ in relation to group $C$ and vice-vers $a=93 \cdot 8 \pm 2 \cdot 2$; for group $A$ in relation to group $B$ and vice-versa $=106 \cdot 6 \pm 3 \cdot 4$; for group $C=32 \cdot 1 \pm 2 \cdot 0$, for group 
$C$ in relation to group $B$ and vice-versa $=133.2 \pm 3.6$ and finally for group $\mathrm{B}=58 \cdot 1 \pm 4 \cdot 0$.

In all cases the inter-group differences are much larger than the intragroup differences and there can be little doubt about the distinctness of three groups of males.

TABLE 2

A calculating sheet to illustrate the method of differential diagnosis

\begin{tabular}{cccc} 
& \multicolumn{3}{c}{ Number of offspring } \\
\cline { 2 - 4 } Batch & Male & Male & Male \\
1 & No. 1 & No. 2 & No. 3 \\
2 & 430 & 446 & 397 \\
3 & 303 & 263 & 298 \\
4 & 244 & 170 & 132 \\
5 & 118 & - & 100 \\
49 & 13 & 34
\end{tabular}

\begin{tabular}{ccc}
\multicolumn{3}{c}{ Differences } \\
\hline $1 / 2$ & $1 / 3$ & $2 / 3$ \\
16 & 33 & 49 \\
40 & 5 & 35 \\
74 & 112 & 38 \\
118 & 18 & 100 \\
36 & 15 & 21 \\
56.8 & 36.6 & 48.6
\end{tabular}

Mean difference

Correct arrangement

\begin{tabular}{cccc}
\multicolumn{3}{c}{ First arrangement } \\
1 & - & 2 & 3 \\
2 & 56.8 & 56.8 & 36.6 \\
3 & 36.6 & 48.6 & -
\end{tabular}

\begin{tabular}{|c|c|c|c|}
\hline & \multicolumn{3}{|c|}{ Correct arrangement } \\
\hline & 1 & 3 & 2 \\
\hline 1 & - & $36 \cdot 6$ & $56 \cdot 8$ \\
\hline 3 & $36 \cdot 6$ & - & $48 \cdot 6$ \\
\hline 2 & $56 \cdot 8$ & $48 \cdot 6$ & - \\
\hline
\end{tabular}

In this example the correct arrangement represented as a diagram (on which only the smallest differences were marked) would have black squares at the intersections: 1/1, 3/3, $2 / 2$ and $1 / 3,3 / 1,2 / 3$; the intersections $1 / 2,3 / 2$ and $2 / 1$ would appear as blank spaces.

\section{Discussion}

The results lead to the conclusion that in the present material of Drosophila melanogaster there are natural differences in the course of spermatogenesis.

In Stromnaess and Kvelland's (1962) material of Drosophila melanogaster the males of the $y s c^{8} Y$ stock were also not uniform in their production. Among them two distinct groups can be defined according to the number of offspring produced in the first and in the second batch. The ratio of these values is 1.18 in one and 0.65 in the other group. Comparable ratios for group $\mathrm{A}, \mathrm{B}$ and $\mathrm{C}$ are: $1.38,1.01$ and 0.73 resp., indicating that there are analogous differences among other Drosophila melanogaster males.

Males of type $\mathrm{C}$ evidently have a small initial supply of spermatozoa and quickly use it up in few matings. There is a gap of temporary sterility between the first lot of sperm and the next. These males may thus reach early stages of spermatogenesis in a shorter time than others. This can be due to a prolonged period of differentiation of spermatids and/or to a poor supply of spermatocytes ready for meiosis, which in turn may be caused by longer spermatogonial cell cycles. In support of the first possibility is the delayed maximum which occurs in the second batch, and of the second is the temporary sterility which appears on the 7th to 8th day probably corresponding to the spermatocyte stage.

Males of type $\mathbf{B}$ have a large initial supply and equal distribution of 
spermatozoa. The transition from one to the next stage of spermatogenesis will be more gradual than in the males of type $\mathrm{C}$. The course of spermatogenesis in the males of group $B$ is rapid and steady. The spermatogonial and meiotic cell cycles as well as cell differentiations are running in such a way that there is an almost constant supply of spermatozoa ready for transfer.

The behaviour of the males of type A can be perhaps explained by assuming shorter periods of differentiation and longer cell cycles.

Males which in the limited period of 10 days produced large number of offspring had spermatogenetic cell cycles and cell differentiations of short duration. Short cell cycles and differentiations include also short sensitive stages resulting in a low level of X-ray mutagenesis. Such males corresponding to the type $B$ males are expected to be low sensitivity males. On the other hand males which produced smaller number of offspring had spermatogenetic cell cycles and cell differentiations of longer duration. Any extension of the duration of spermatogenesis naturally involves also the extension of the sensitive stage, therefore the males of low productivity are expected to show more sensitivity to the action of X-rays. Males of type $\mathrm{G}$ probably represent such highly sensitive males.

The question could be asked where to look for a phase in which cell becomes highly sensitive to X-rays. According to Sobels' (1965) review the spermatogenetic sensitivity to X-rays is highest in spermatids and late spermatocytes, then in early spermatocytes, then in the first day sperm, second day sperm and finally, the lowest in spermatogonia. Sobels points out the arbitrary and tentative character of this arrangement. For structural changes Slizynska (loc. cit.) has shown that spermatogonial cells, if sensitive, present even greater response to X-rays than spermatids. From her results it follows that after the irradiation spermatids show few chromosome breaks per cell in many cells, while after the irradiation of spermatogonia there are many breaks per cell in few cells. Spermatids are non-dividing cells and most of them undergo the same process of differentiation. Their sensitivity is thus related to cell differentiation and clearly not to cell division. On the other hand spermatogonia are mostly in the interphase, few of them going through mitotic divisions followed by differentiation into spermatocytes. Thus spermatogonial cells may have the sensivity related rather to cell division.

During cell division all chromosomes become stainable and all genes inactivated, while during cell differentiation some parts of chromosomes become stainable and some genes inactivated. It could be suggested that sensitivity to X-rays is proportional to the size of stainable and inactivated regions.

\section{Summary}

1. Drosophila melanogaster males differ in the output of their spermatogenesis during the first 10 days of their life.

2. There are three types of male: A - start with high yield of offspring in the first matings followed by a steady, gradual decline, B-start with high yield followed by a very slow decline and almost unchanged level of production, and $\mathrm{C}$-which have the highest yield in the second batch of females followed by a rapid decline.

3 . These differences are naturally related to the variation in the duration of cell cycles and cell differentiations of spermatogenesis. 
4. Prolongation of cell cycles includes also prolongation of the sensitive stage which may result in more effective mutagenic action of X-rays. Thus less prolific males are apt to be more responsive to the $\mathrm{X}$-rays.

Acknowledgments.-My thanks are due to Miss Patricia Rutherford for technical help in the course of the experiment.

\section{REFERENGES}

CZekanowski, J. 1913. Zarys metod statystycznych. Prace Warsz. Tow. Nauk. No. 5. Warszawa 1913.

SLIZYNSKA, H. 1963. Heterogeneity among spermatogonia of Drosophila melanogaster males. Gen. Res., 4, 446-456.

soBeLs, F. H. 1965. Radiosensitivity and repair in different germ cell stages of Drosophila. Genetics Today, 2, 236-255.

STROMNAESS, O., AND KVELL.AND, I. 1962. Sexual activity of Drosophila melanogaster males. Hereditas, 48, 442-470. 